\title{
Rice Herbicides for Cotton + Soybean Intercropping
}

\author{
A. Ravinder Raju* \\ Central Institute for Non Bt cotton Research, P.B.No.2 Shankarnagar Post office, \\ Nagpur 440010 (M.S.), India
}

*Corresponding author

\begin{abstract}
A B S T R A C T
A field experiment was conducted at Central Institute for Cotton Research Farm, Nagpur for three years in medium deep clay loam soil. The experiment was laid out in RBD design with three replications consist of three herbicide based weed management packages in

\section{Keywords}

Butachlor, Intercropping, Oxyflurofen,

Pendimethalin, Rice herbicides

\section{Article Info}

Accepted:

26 July 2018

Available Online:

10 August 2018 intercropping of non Bt cotton + soybean and two sole crops. Non Bt cotton+ soybean intercropping reduced seed cotton yields considerably in a year of very $640 \mathrm{~mm}$ higher rainfall over normal caused over growth of soybean. In second year also the seed cotton yields were low due to very high incidence of Helicoverpa armigera caused non recovery of intercropped cotton. The intercropped soybean grain yields were non significantly influenced in all the years by herbicide treatments and intercropping. The conventional herbicide package consists of Pendimethalin $0.75 \mathrm{~kg}$ a. i. ha ${ }^{-1}$ as PPI along with two interculture operations at 21 and 42 DAS was found to be most efficient. However, most economical and relatively efficient rice herbicides were Butachlor $1.0 \mathrm{~kg}$ as PPI or Oxyflurofen $0.1 \mathrm{~kg}$ a. i. ha ${ }^{-1}$ as pre emergence with two hoeings at 30 and 45 DAS and one hand weeding at 21 DAS to remove any herbicide resistant broad leaf weeds. All three herbicide packages tested were efficient economical and easily adoptable due to their local availability and all ready being used in rice ecosystem. Subsequently results were validated and demonstrated in $\mathrm{Bt}$ and non Bt hybrid cotton in station, on farm trials and farmers fields in the last 15 years.
\end{abstract}

\section{Introduction}

Non Bt cotton crop is widely spaced at $90 \mathrm{~cm}$ row spacing under rainfed vertisols of central India. This wide space allows luxuriant weed growth in monsoon season that can be used for raising a short duration leguminous intercrops, which can suppress the weeds (Bonde and Raju 1996; Raju et al., 2013; Raju and Thakare, 2014). Non Bt cotton + soybean (1: 1) intercropping offers benefits such as insurance against crop failure due to aberrations in rainfall or out breaks of pest incidence with better profitability unit area ${ }^{-1}$ and time $^{-1}$. Besides these the intercrop suppresses broad leaved weeds and root nodules contribute to soil fertility improvement. Screening of suitable varieties for intercropping in non Bt cotton at Central Institute for Cotton Research, Nagpur found non Bt cotton genotypes LRA 5166, hybrid-6 and soybean varieties Pusa-16, PKV-1, PK472, TAS-40 and Punjab-1 were found suitable (Anon, 1994). Although non Bt cotton 
and soybean were the major crops of this area and their intercropping is accepted on a limited scale due to inconvenience in crosswise intercultural operations. The other reasons for non-acceptance of earlier recommended intercrops like green and black gram is catching intercrops in post September rains. Nimbole (1990) suggested to use Fluchloralin a, dinitro aniline herbicide in intercropping of non $\mathrm{Bt}$ hybrid cotton. The primary objective of this experiment was to adopt and develop the rice herbicides based low cost integrated weed management package for intercropped non Bt cotton. Therefore, a field experiment was planned to study the effective and economic rice herbicide based integrated weed management package for non $\mathrm{Bt}$ cotton + soybean intercropping.

\section{Materials and Methods}

The field experiment was planted in monsoon season with 3 herbicides and two earthingups based integrated weed management treatments of non Bt cotton + soybean row intercropping and three controls i.e. Farmer's practice and two sole crops in R.B.D. design with three replications in medium deep vertisols. The intercropped non Bt cotton was planted at $90 \mathrm{x}$ $15 \mathrm{~cm}$ and soybean at $30 \mathrm{~cm}$ in rows, sole crop treatments of non Bt cotton was planted at 60 x $30 \mathrm{~cm}$ and soybean at $45 \times 15 \mathrm{~cm}$ as per recommendation. The fertilizers were applied to both the crops as per their respective recommended doses of fertilizers (R.D.F.) i. e. 60:30:30 and 20: 50: $0 \mathrm{~kg} \mathrm{~N}, \mathrm{P}_{2} \mathrm{O}_{5}$ and $\mathrm{K}_{2} \mathrm{O}$ respectively in proportion to their population for non Bt cotton and soybean crops, whereas for intercropping of soybean $60 \%$ RDF was supplemented in addition to $100 \%$ RDF for non $\mathrm{Bt}$ cotton. Intercultural operations were performed in rows in one direction only. The treatment details are $\mathrm{T}_{1}$ : Pendimethalin herbicide@0.75 kg a. i. ha ${ }^{-1}$ as pre plant incorporation $(\mathrm{PPI})+$ two interculture operations at 21 and 42 DAS; $\mathrm{T}_{2}$ : Two intercultural operations at 21, 42 DAS followed by two earthingup operations; $\mathrm{T}_{3}$ : Two intercultural operations at 21, 42 DAS followed by one hand weeding along with earthingup operation after first interculture operation; $\mathrm{T}_{4}$ : Two intercultural operations at 21, 42 DAS followed by two hand weedings at 21 and 42 DAS (check); $\mathrm{T}_{5}$ : Oxyflurofen 0.1 $\mathrm{kg}$ a. i. $\mathrm{ha}^{-1}$ as pre emergence with 2 intercultural operations at 30 and 45 DAS followed by one hand weeding at 21 DAS to remove resistant broad leaved weeds; $\mathrm{T}_{6}$ : Butachlor $1.0 \mathrm{~kg}$ a. i. ha ${ }^{-1}$ as PPI with two intercultural operations at 30 and 45 DAS followed by one hand weeding at 21 DAS to remove resistant broad leaved weeds (one year); $\mathrm{T}_{7}$ : Sole crop of non Bt cotton C. var. LRA 5166 with recommended cultural weed management i.e. Three intercultural operations at 21, 42 and 63 DAS followed by two hand weedings at 21 and 42 DAS ; $\mathrm{T}_{8}$ : Sole crop of soybean C. var. Punjab-1 with two intercultural operations at 21, 42 DAS followed by two hand weedings at 21 and 42 DAS. The herbicides tolerance was evaluated in pot trial for non $\mathrm{Bt}$ cotton and soybean before starting of field trial. The weed populations were converted to $\log \mathrm{V}(\mathrm{x}+21)$ before statistical analysis.

\section{Results and Discussion}

\section{Weed density $\mathbf{M}^{2}$}

The common weed flora of the experimental site were Phylanthus niruri, Celocia argentia, Convolvulus arvensis, Euphorbea geniculata, E. macrocephala, Cynotis auxillaris, Merremia emarginata, Panicum repense, Thelepogon elegans, Digitaria ciliaris and Echinocloa colonum were observed. In first season, the weed management treatments were significantly influenced on Di cots and total weed population (Table.1). Non Bt cotton + soybean intercropping has successfully 
suppressed weeds compared to both the sole crops. The soybean as sole crop had highest dicot weed population at the time of first interculture. Oxyflurofen herbicide had significantly lowest weed density while pendimethalin had statistically similar weed populations to that of intercultures along with hand weedings.

After the second interculture only total weed population was influenced and no distinction within sole and intercrops was observed probably due to weed suppression by soybean canopy coverage and residual influence of herbicide treatments. Farmer's practice of three intercultures and hand weedings had significantly lowest weed density, which was at par with oxyflurofen herbicides as pre emergence application.

In second season, at the time of first interculture oxyflurofen as pre em application had significantly lowest weed density (Table. 2) compared to normal cultural practices treatment. Mono cot weeds population was reduced by $90 \%$ and di cots by $50 \%$ by Oxyflurofen pre emergence spray without harming cotton or soybean. Similarly, the differences within treatments $T_{3}, T_{4}$ and $T_{7}, T_{8}$ were at par. There was no distinction between sole and inter crops for weed density due 1.5 times excess rains received and intercropped soybean over grown and suppress the weeds.

After second interculture the dicots population reduced to minimal due to interculture effect but mono cots population remained as such and therefore treatment differences were reduced to non-significant level. This probably due to faster leaf expansion of soybean under cloudy weather and higher rainfall lead to early canopy closing. In the final year monsoon season weed density of monocot weeds were non significantly removed by $1^{\text {st }}$ interculture (Table 3) and hand weeding in sole and inter crops.
Butachlor as PPI application was relatively more efficient in controlling dicot weeds compared to oxyflurofen as pre em application and rest were at par. Among total weed densities treatments $T_{5}$ and $T_{6}$ were at par while they had significantly higher weed density patches than normal intercultures and hand weedings $\left(\mathrm{T}_{4}\right)$.

At the time of second interculture and hand weeding monocot weeds were non significantly influenced by sole and intercrops (Table. 4). Similarly was with dicot weeds by second interculture except treatments $T_{5}$ and $\mathrm{T}_{6}$ i.e. rice herbicides had higher dicot weeds. At the time of $3^{\text {rd }}$. interculture after harvesting of intercrop hand weeding significantly removed (Table.5) monocot weeds which were higher in sole crops, treatment $\mathrm{T}_{6}$ had significantly higher monocot weeds than farmer's practice $\left(T_{4}\right)$, while rice herbicides $T_{5}$ and $\mathrm{T}_{6}$ were statistically at par. Therefore the butachlor efficacy needs to be confirmed with further studies.

\section{Weed biomass}

Weed biomass was non significantly differing (Table. 6) by sole and intercrops. Pendimethalin $\left(\mathrm{T}_{1}\right)$ and farmer's practice along with earthingups $\left(\mathrm{T}_{3}\right)$ had significantly lower biomass than treatment farmer's practice alone $\left(\mathrm{T}_{4}\right)$.

Additional hand weeding at 21 DAS in treatment $T_{3}$ proved its effectiveness in rainy season with continuous rains. On $1^{\text {st }}$ hand weeding the treatment differences failed to influence on weed biomass. At the time of $2^{\text {nd }}$ interculture and hand weeding treatment along with Pendimethalin in treatment $T_{1}$ had higher weeds because of dry weather subsequently to its application. Rice herbicide treatments Butachlor $\left(\mathrm{T}_{5}\right)$ and oxyflurofen $\left(\mathrm{T}_{6}\right)$ had lowest weed biomass among all the weed management treatments. 
Table.1 Weed density $\mathrm{M}^{2}$ by hand weeding in first season

\begin{tabular}{|c|c|c|c|c|c|c|}
\hline \multirow[t]{2}{*}{ T. No } & \multicolumn{3}{|c|}{ hand weeding 22 July } & \multicolumn{3}{|c|}{ hand weeding $7^{\text {th }}$ September } \\
\hline & $\begin{array}{l}\text { Mono } \\
\text { cots }\end{array}$ & Di cots & Total & $\begin{array}{l}\text { Mono } \\
\text { cots }\end{array}$ & Di cots & Total \\
\hline $\begin{array}{l}\mathrm{T}_{1} \text { : Pendimethalin@ } 0.75 \mathrm{~kg} \text { a. i. ha }{ }^{-1} \text { as PPI + } \\
\text { two interculture operations at } 21 \text { and } 42 \mathrm{DAS} \text {. }\end{array}$ & & $\begin{array}{c}1.33 \\
(0.6743)\end{array}$ & $\begin{array}{c}1.33 \\
(0.6743)\end{array}$ & & & \\
\hline $\begin{array}{l}\mathrm{T}_{2} \text { : Two intercultural operations at } 21,42 \text { DAS } \\
\text { followed by two earthingup operations. }\end{array}$ & $\begin{array}{c}0.33 \\
(0.6645)\end{array}$ & $\begin{array}{c}2.16 \\
(0.6823)\end{array}$ & $\begin{array}{c}2.5 \\
(0.6854)\end{array}$ & & & \\
\hline $\begin{array}{l}\mathrm{T}_{3} \text { : Two intercultural operations at } 21,42 \text { DAS } \\
\text { followed by one hand weeding alongwith } \\
\text { earthingup operation. }\end{array}$ & $\begin{array}{c}0.33 \\
(0.6645)\end{array}$ & $\begin{array}{c}1.83 \\
(0.6792)\end{array}$ & $\begin{array}{c}2.16 \\
(0.6624)\end{array}$ & & & \\
\hline $\begin{array}{l}\mathrm{T}_{4} \text { : Two intercultural operations at } 21,42 \mathrm{DAS} \\
\text { followed by two hand weedings at } 21 \text { and } 42 \\
\text { DAS. }\end{array}$ & $\begin{array}{c}0.66 \\
(0.6678)\end{array}$ & $\begin{array}{c}2.0 \\
(0.6805)\end{array}$ & $\begin{array}{c}2.66 \\
(0.6838)\end{array}$ & $\begin{array}{c}0.5 \\
(0.6662)\end{array}$ & $\begin{array}{c}1.83 \\
(0.6792)\end{array}$ & $\begin{array}{c}2.33 \\
(0.6774)\end{array}$ \\
\hline $\begin{array}{l}\mathrm{T}_{5} \text { : Oxyflurofen } 0.1 \mathrm{~kg}^{\mathrm{a}} \text { i. ha }{ }^{-1} \text { as pre emergence } \\
\text { with } 2 \text { intercultural operations at } 30 \text { and } 45 \text { DAS } \\
\text { followed by one hand weeding at } 21 \text { DAS. }\end{array}$ & & $\begin{array}{c}0.16 \\
(0.6628)\end{array}$ & $\begin{array}{c}0.16 \\
(0.6628)\end{array}$ & $\begin{array}{c}0.33 \\
(0.6645)\end{array}$ & $\begin{array}{c}2.33 \\
(0.6839)\end{array}$ & $\begin{array}{c}2.66 \\
(0.6787)\end{array}$ \\
\hline $\begin{array}{l}\mathrm{T}_{7} \text { : Sole crop of non Bt cotton C. var. LRA } 5166 \\
\text { with recommended cultural weed management } \\
\text { i.e. Three intercultural operations at } 21,42 \text { and } \\
63 \text { DAS followed by two hand weedings at } 21 \\
\text { and } 42 \text { DAS. }\end{array}$ & $\begin{array}{c}0.33 \\
(0.6645)\end{array}$ & $\begin{array}{c}5.33 \\
(0.7095)\end{array}$ & $\begin{array}{c}5.66 \\
(0.7120)\end{array}$ & $\begin{array}{c}2.33 \\
(0.6774)\end{array}$ & $\begin{array}{c}2.33 \\
(0.6774)\end{array}$ & $\begin{array}{c}4.68 \\
(0.6973)\end{array}$ \\
\hline $\begin{array}{l}\mathrm{T}_{8} \text { : Sole crop of soybean C. var. Punjab- } 1 \text { with } \\
\text { two intercultural operations at } 21,42 \text { DAS } \\
\text { followed by two hand weedings at } 21 \text { and } 42 \\
\text { DAS. }\end{array}$ & & $\begin{array}{c}9.83 \\
(0.7438)\end{array}$ & $\begin{array}{c}9.83 \\
(0.7438)\end{array}$ & $\begin{array}{c}1.33 \\
(0.6743)\end{array}$ & $2.66(0.6787)$ & $\begin{array}{c}4.0 \\
(0.6901)\end{array}$ \\
\hline $\mathrm{SED} \pm 5 \%$ & 0.0025 & & & 0.0275 & 0.0112 & \\
\hline $\mathrm{CD} \pm 5 \%$ & NS & 0.0214 & 0.0201 & NS & NS & 0.0189 \\
\hline
\end{tabular}

Table. 2 Weed density $\mathrm{M}^{2}$ by hand weeding in second season

\begin{tabular}{|c|c|c|c|c|c|c|}
\hline \multirow[t]{2}{*}{ T.No } & \multicolumn{3}{|c|}{$30^{\text {th }}$ July } & \multicolumn{3}{|c|}{ 31 ${ }^{\text {st }}$ August. } \\
\hline & $\begin{array}{l}\text { Mono } \\
\text { cots }\end{array}$ & Di cots & Total & $\begin{array}{l}\text { Mono } \\
\text { cots }\end{array}$ & Di cots & Total \\
\hline \multicolumn{7}{|l|}{$\begin{array}{l}\mathrm{T}_{1} \text { : Pendimethalin @ } 0.75 \mathrm{~kg} \text { a. i. ha }{ }^{-1} \text { as PPI }+ \text { two } \\
\text { interculture operations at } 21 \text { and } 42 \mathrm{DAS} \text {. }\end{array}$} \\
\hline \multicolumn{7}{|l|}{$\begin{array}{l}\mathrm{T}_{2} \text { : Two intercultural operations at } 21,42 \text { DAS followed by } \\
\text { two earthingup operations. }\end{array}$} \\
\hline $\begin{array}{l}\mathrm{T}_{3} \text { : Two intercultural operations at } 21,42 \mathrm{DAS} \text { followed by } \\
\text { one hand weeding alongwith earthingup operation. }\end{array}$ & $\begin{array}{c}5.5 \\
(0.710)\end{array}$ & $\begin{array}{c}8.16 \\
(0.731)\end{array}$ & $\begin{array}{c}13.66 \\
(0.766)\end{array}$ & & & \\
\hline $\begin{array}{l}\mathrm{T}_{4} \text { : Two intercultural operations at } 21,42 \text { DAS followed by } \\
\text { two hand weedings at } 21 \text { and } 42 \text { DAS. }\end{array}$ & $\begin{array}{l}10.16 \\
(0.741)\end{array}$ & $\begin{array}{l}10.33 \\
(0.744)\end{array}$ & $\begin{array}{c}20.5 \\
(0.808)\end{array}$ & $\begin{array}{c}10.17 \\
(0.743)\end{array}$ & $\begin{array}{c}2.83 \\
(0.688)\end{array}$ & $\begin{array}{c}13.0 \\
(0.761)\end{array}$ \\
\hline $\begin{array}{l}\mathrm{T}_{5} \text { : Oxyflurofen } 0.1 \mathrm{~kg} \text { a. i. ha }{ }^{-1} \text { as pre emergence with } 2 \\
\text { intercultural operations at } 30 \text { and } 45 \text { DAS followed by one } \\
\text { hand weeding at } 21 \text { DAS. }\end{array}$ & $\begin{array}{c}1.0 \\
(0.670)\end{array}$ & $\begin{array}{c}5.66 \\
(0.709)\end{array}$ & $\begin{array}{c}6.66 \\
(0.717)\end{array}$ & $\begin{array}{c}9.17 \\
(0.738)\end{array}$ & $\begin{array}{c}2.33 \\
(0.683)\end{array}$ & $\begin{array}{c}11.5 \\
(0.755)\end{array}$ \\
\hline $\begin{array}{l}\mathrm{T}_{7} \text { : Sole crop of non Bt cotton C. var. LRA } 5166 \text { with } \\
\text { recommended cultural weed management i.e. Three } \\
\text { intercultural operations at } 21,42 \text { and } 63 \text { DAS followed by } \\
\text { two hand weedings at } 21 \text { and } 42 \text { DAS. }\end{array}$ & $\begin{array}{c}7.0 \\
(0.719)\end{array}$ & $\begin{array}{c}12.0 \\
(0.758)\end{array}$ & $\begin{array}{c}22.33 \\
(0.817)\end{array}$ & $\begin{array}{c}6.17 \\
(0.716)\end{array}$ & $\begin{array}{c}4.5 \\
(0.703)\end{array}$ & $\begin{array}{c}10.7 \\
(0.749)\end{array}$ \\
\hline $\begin{array}{l}\mathrm{T}_{8} \text { : Sole crop of soybean C. var. Punjab- } 1 \text { with two } \\
\text { intercultural operations at } 21,42 \text { DAS followed by two } \\
\text { hand weedings at } 21 \text { and } 42 \text { DAS. }\end{array}$ & $\begin{array}{c}7.16 \\
(0.723)\end{array}$ & $\begin{array}{l}10.16 \\
(0.746)\end{array}$ & $\begin{array}{c}17.33 \\
(0.791)\end{array}$ & $\begin{array}{c}5.33 \\
(0.710)\end{array}$ & $\begin{array}{c}6.17 \\
(0.715)\end{array}$ & $\begin{array}{c}11.5 \\
(0.755)\end{array}$ \\
\hline $\mathrm{SED} \pm 5 \%$ & 0.022 & 0.001 & & 0.014 & 0.040 & 0.015 \\
\hline $\mathrm{CD} \pm 5 \%$ & NS & NS & 0.066 & NS & NS & NS \\
\hline
\end{tabular}


Table.3 Weed density $\mathrm{M}^{2}$ on $26^{\text {th }}$ July third season

\begin{tabular}{|c|c|c|c|c|c|c|}
\hline \multirow[t]{2}{*}{ T.No } & \multicolumn{3}{|c|}{ Interculture } & \multicolumn{3}{|c|}{ Hand weeding } \\
\hline & $\begin{array}{l}\text { Mono } \\
\text { cots }\end{array}$ & Di cots & Total & Monocots & Di cots & Total \\
\hline $\begin{array}{l}\mathrm{T}_{1} \text { : Pendimethalin @ } 0.75 \mathrm{~kg} \text { a. i. ha } \mathrm{ha}^{-1} \text { as PPI + two } \\
\text { interculture operations at } 21 \text { and } 42 \mathrm{DAS} \text {. }\end{array}$ & & & & $\begin{array}{c}0.99 \\
(0.518)\end{array}$ & $\begin{array}{c}3.0 \\
(0.689)\end{array}$ & $\begin{array}{c}3.99 \\
(0.698)\end{array}$ \\
\hline $\begin{array}{l}\mathrm{T}_{2} \text { : Two intercultural operations at } 21,42 \text { DAS followed } \\
\text { by two earthingup operations. }\end{array}$ & $\begin{array}{c}1.78 \\
(0.698)\end{array}$ & $\begin{array}{c}2.44 \\
(0.684)\end{array}$ & $\begin{array}{c}4.22 \\
(0.700)\end{array}$ & $\begin{array}{c}1.33 \\
(0.674)\end{array}$ & $\begin{array}{c}1.84 \\
(0.672)\end{array}$ & $\begin{array}{c}2.10 \\
(0.681)\end{array}$ \\
\hline $\begin{array}{l}\mathrm{T}_{3} \text { : Two intercultural operations at } 21,42 \text { DAS followed } \\
\text { by one hand weeding alongwith earthingup operation. }\end{array}$ & $\begin{array}{c}2.78 \\
(0.687)\end{array}$ & $\begin{array}{l}1.55 \\
(0.676)\end{array}$ & $\begin{array}{c}4.33 \\
(0.701)\end{array}$ & $\begin{array}{c}2.0 \\
(0.680)\end{array}$ & $\begin{array}{c}1.55 \\
(0.673)\end{array}$ & $\begin{array}{c}3.54 \\
(0.694)\end{array}$ \\
\hline $\begin{array}{l}\mathrm{T}_{4} \text { : Two intercultural operations at } 21,42 \text { DAS followed } \\
\text { by two hand weedings at } 21 \text { and } 42 \text { DAS. }\end{array}$ & $\begin{array}{l}1.67 \\
(0.677)\end{array}$ & $\begin{array}{c}2.44 \\
(0.684)\end{array}$ & $\begin{array}{c}4.11 \\
(0.699)\end{array}$ & $\begin{array}{c}1.55 \\
(0.674)\end{array}$ & $\begin{array}{c}1.89 \\
(0.679)\end{array}$ & $\begin{array}{c}3.44 \\
(0.694)\end{array}$ \\
\hline $\begin{array}{l}\mathrm{T}_{5} \text { : Oxyflurofen } 0.1 \mathrm{~kg} \text { a. i. ha }{ }^{-1} \text { as pre emergence with } 2 \\
\text { intercultural operations at } 30 \text { and } 45 \text { DAS followed by } \\
\text { one hand weeding at } 21 \text { DAS. }\end{array}$ & $\begin{array}{c}3.22 \\
(0.691)\end{array}$ & $\begin{array}{c}5.44 \\
(0.711)\end{array}$ & $\begin{array}{c}8.66 \\
(0.735)\end{array}$ & $\begin{array}{c}0.83 \\
(0.669)\end{array}$ & $\begin{array}{c}1.66 \\
(0.677)\end{array}$ & $\begin{array}{c}3.88 \\
(0.697)\end{array}$ \\
\hline $\begin{array}{l}\mathrm{T}_{6} \text { : Butachlor } 1.0 \mathrm{~kg} \text { a. i. } \mathrm{ha}^{-1} \text { as PPI with two } \\
\text { intercultural operations at } 30 \text { and } 45 \text { DAS followed by } \\
\text { one hand weeding at } 21 \mathrm{DAS}\end{array}$ & $\begin{array}{c}2.44 \\
(0.684)\end{array}$ & $\begin{array}{c}4.78 \\
(0.705)\end{array}$ & $\begin{array}{c}7.22 \\
(0.725)\end{array}$ & $\begin{array}{c}1.99 \\
(0.680)\end{array}$ & $\begin{array}{c}1.66 \\
(0.677)\end{array}$ & $\begin{array}{c}3.88 \\
(0.697)\end{array}$ \\
\hline $\begin{array}{l}\mathrm{T}_{7} \text { : Sole crop of non Bt cotton C. var. LRA } 5166 \text { with } \\
\text { recommended cultural weed management i.e. Three } \\
\text { intercultural operations at } 21,42 \text { and } 63 \text { DAS followed } \\
\text { by two hand weedings at } 21 \text { and } 42 \text { DAS. }\end{array}$ & $\begin{array}{c}1.56 \\
(0.676)\end{array}$ & $\begin{array}{c}2.0 \\
(0.680)\end{array}$ & $\begin{array}{c}3.55 \\
(0.695)\end{array}$ & $\begin{array}{c}2.0 \\
(0.680)\end{array}$ & $\begin{array}{c}1.67 \\
(0.677)\end{array}$ & $\begin{array}{c}3.66 \\
(0.696)\end{array}$ \\
\hline $\begin{array}{l}\mathrm{T}_{8} \text { : Sole crop of soybean C. var. Punjab- } 1 \text { with two } \\
\text { intercultural operations at } 21,42 \text { DAS followed by two } \\
\text { hand weedings at } 21 \text { and } 42 \text { DAS. }\end{array}$ & $\begin{array}{c}1.56 \\
(0.676)\end{array}$ & $\begin{array}{c}2.0 \\
(0.680)\end{array}$ & $\begin{array}{c}3.55 \\
(0.690)\end{array}$ & $\begin{array}{c}2.89 \\
(0.688)\end{array}$ & $\begin{array}{c}1.44 \\
(0.675)\end{array}$ & $\begin{array}{c}4.3 \\
(0.701)\end{array}$ \\
\hline $\mathrm{SED} \pm 5 \%$ & 0.059 & & & 0.054 & 0.004 & 0.062 \\
\hline $\mathrm{CD} \pm 5 \%$ & NS & 0.016 & 0.021 & NS & NS & NS \\
\hline
\end{tabular}

Table. 4 Weed density $\mathrm{M}^{2}$ on $9^{\text {th }}$ August third season

\begin{tabular}{|c|c|c|c|c|c|c|}
\hline \multirow[t]{2}{*}{ T.No } & \multicolumn{3}{|c|}{ Interculture } & \multicolumn{3}{|c|}{ Hand weeding } \\
\hline & $\begin{array}{l}\text { Mono } \\
\text { cots }\end{array}$ & Di cots & Total & $\begin{array}{l}\text { Mono } \\
\text { cots }\end{array}$ & Di cots & Total \\
\hline $\begin{array}{l}\mathrm{T}_{1} \text { : Pendimethalin @ } 0.75 \mathrm{~kg} \text { a. i. } \mathrm{ha}^{-1} \text { as PPI + two } \\
\text { interculture operations at } 21 \text { and } 42 \mathrm{DAS} \text {. }\end{array}$ & $\begin{array}{l}1.77 \\
(0.679)\end{array}$ & $\begin{array}{c}1.66 \\
(0.674)\end{array}$ & $\begin{array}{c}3.43 \\
(0.691)\end{array}$ & $\begin{array}{c}2.22 \\
(0.681)\end{array}$ & $\begin{array}{c}5.22 \\
(0.708)\end{array}$ & $\begin{array}{c}7.60 \\
(0.728)\end{array}$ \\
\hline $\begin{array}{l}\mathrm{T}_{2} \text { : Two intercultural operations at } 21,42 \mathrm{DAS} \text { followed } \\
\text { by two earthingup operations. }\end{array}$ & $\begin{array}{c}1.66 \\
(0.678)\end{array}$ & $\begin{array}{c}0.55 \\
(0.666)\end{array}$ & $\begin{array}{c}2.21 \\
(0.678)\end{array}$ & $\begin{array}{c}2.33 \\
(0.683)\end{array}$ & $\begin{array}{c}2.66 \\
(0.687)\end{array}$ & $\begin{array}{c}2.99 \\
(0.699)\end{array}$ \\
\hline $\begin{array}{l}\mathrm{T}_{3} \text { : Two intercultural operations at } 21,42 \mathrm{DAS} \text { followed } \\
\text { by one hand weeding along with earthingup operation. }\end{array}$ & $\begin{array}{c}1.33 \\
(0.679)\end{array}$ & $\begin{array}{c}1.22 \\
(0.673)\end{array}$ & $\begin{array}{c}2.55 \\
(0.681)\end{array}$ & $\begin{array}{c}2.11 \\
(0.681)\end{array}$ & $\begin{array}{c}2.55 \\
(0.684)\end{array}$ & $\begin{array}{c}4.66 \\
(0.703)\end{array}$ \\
\hline $\begin{array}{l}\mathrm{T}_{4} \text { : Two intercultural operations at } 21,42 \text { DAS followed } \\
\text { by two hand weedings at } 21 \text { and } 42 \text { DAS. }\end{array}$ & $\begin{array}{c}1.88 \\
(0.680)\end{array}$ & $\begin{array}{c}0.88 \\
(0.670)\end{array}$ & $\begin{array}{c}2.77 \\
(0.682)\end{array}$ & $\begin{array}{c}2.66 \\
(0.683)\end{array}$ & $\begin{array}{c}3.66 \\
(0.695)\end{array}$ & $\begin{array}{c}6.32 \\
(0.709)\end{array}$ \\
\hline $\begin{array}{l}\mathrm{T}_{5} \text { : Oxyflurofen } 0.1 \mathrm{~kg} \text { a. i. ha }{ }^{-1} \text { as pre emergence with } 2 \\
\text { intercultural operations at } 30 \text { and } 45 \text { DAS followed by } \\
\text { one hand weeding at } 21 \text { DAS. }\end{array}$ & $\begin{array}{c}1.44 \\
(0.676)\end{array}$ & $\begin{array}{c}2.88 \\
(0.688)\end{array}$ & $\begin{array}{c}4.33 \\
(0.696)\end{array}$ & $\begin{array}{c}0.86 \\
(0.669)\end{array}$ & $\begin{array}{c}2.55 \\
(0.685)\end{array}$ & $\begin{array}{c}3.43 \\
(0.691)\end{array}$ \\
\hline $\begin{array}{l}\mathrm{T}_{6} \text { : Butachlor } 1.0 \mathrm{~kg} \mathrm{a} \text {. i. } \mathrm{ha}^{-1} \text { as PPI with two } \\
\text { intercultural operations at } 30 \text { and } 45 \text { DAS followed by } \\
\text { one hand weeding at } 21 \mathrm{DAS}\end{array}$ & $\begin{array}{c}2.77 \\
(0.688)\end{array}$ & $\begin{array}{c}2.55 \\
(0.685)\end{array}$ & $\begin{array}{c}5.32 \\
(0.701)\end{array}$ & $\begin{array}{c}2.44 \\
(0.684)\end{array}$ & $\begin{array}{c}2.22 \\
(0.682)\end{array}$ & $\begin{array}{c}4.66 \\
(0.700)\end{array}$ \\
\hline $\begin{array}{l}\mathrm{T}_{7} \text { : Sole crop of non Bt cotton C. var. LRA } 5166 \text { with } \\
\text { recommended cultural weed management i.e. Three } \\
\text { intercultural operations at } 21,42 \text { and } 63 \text { DAS followed } \\
\text { by two hand weedings at } 21 \text { and } 42 \text { DAS. }\end{array}$ & $\begin{array}{c}1.88 \\
(0.680)\end{array}$ & $\begin{array}{c}1.11 \\
(0.672)\end{array}$ & $\begin{array}{c}2.99 \\
(0.682)\end{array}$ & $\begin{array}{c}3.44 \\
(0.693)\end{array}$ & $\begin{array}{c}3.33 \\
(0.692)\end{array}$ & $\begin{array}{c}6.76 \\
(0.705)\end{array}$ \\
\hline $\begin{array}{l}\mathrm{T}_{8} \text { : Sole crop of soybean C. var. Punjab- } 1 \text { with two } \\
\text { intercultural operations at } 21,42 \text { DAS followed by two } \\
\text { hand weedings at } 21 \text { and } 42 \text { DAS. }\end{array}$ & $\begin{array}{c}1.55 \\
(0.678)\end{array}$ & $\begin{array}{c}0.55 \\
(0.666)\end{array}$ & $\begin{array}{c}2.10 \\
(0.677)\end{array}$ & $\begin{array}{c}3.0 \\
(0.689)\end{array}$ & $\begin{array}{c}1.0 \\
(0.671)\end{array}$ & $\begin{array}{c}3.99 \\
(0.682)\end{array}$ \\
\hline $\mathrm{SED} \pm 5 \%$ & 0.003 & & 0.005 & 0.009 & 0.009 & 0.008 \\
\hline $\mathrm{CD} \pm 5 \%$ & NS & 0.012 & NS & NS & NS & NS \\
\hline
\end{tabular}


Table.5 Weed density $\mathrm{M}^{2}$ on $21^{\text {st }}$ October third season

\begin{tabular}{|c|c|c|c|}
\hline \multirow[t]{2}{*}{ T. No } & \multicolumn{3}{|c|}{ Hand weeding } \\
\hline & Mono cots & Di cots & Total \\
\hline $\mathrm{T}_{1}$ : Pendimethalin @ $0.75 \mathrm{~kg}$ a. i. ha ${ }^{-1}$ as PPI + two interculture operations at 21 and $42 \mathrm{DAS}$. & $\begin{array}{c}3.0 \\
(0.680)\end{array}$ & $\begin{array}{c}3.16 \\
(0.690)\end{array}$ & $\begin{array}{c}5.16 \\
(0.702)\end{array}$ \\
\hline $\mathrm{T}_{2}$ : Two intercultural operations at 21,42 DAS followed by two earthingup operations. & $\begin{array}{c}3.16 \\
(0.691)\end{array}$ & $\begin{array}{c}4.66 \\
(0.703)\end{array}$ & $\begin{array}{c}7.83 \\
(0.716)\end{array}$ \\
\hline $\begin{array}{l}\mathrm{T}_{3} \text { : Two intercultural operations at } 21,42 \text { DAS followed by one hand weeding along with earthingup } \\
\text { operation. }\end{array}$ & $\begin{array}{c}2.5 \\
(0.685)\end{array}$ & $\begin{array}{c}3.83 \\
(0.697)\end{array}$ & $\begin{array}{c}6.33 \\
(0.705)\end{array}$ \\
\hline $\mathrm{T}_{4}$ : Two intercultural operations at $21,42 \mathrm{DAS}$ followed by two hand weedings at 21 and $42 \mathrm{DAS}$. & $\begin{array}{c}2.16 \\
(0.682)\end{array}$ & $\begin{array}{c}5.33 \\
(0.709)\end{array}$ & $\begin{array}{c}7.50 \\
(0.720)\end{array}$ \\
\hline $\begin{array}{l}T_{5} \text { : Oxyflurofen } 0.1 \mathrm{~kg} \text { a. i. ha }{ }^{-1} \text { as pre emergence with } 2 \text { intercultural operations at } 30 \text { and } 45 \text { DAS } \\
\text { followed by one hand weeding at } 21 \text { DAS. }\end{array}$ & $\begin{array}{c}5.33 \\
(0.706)\end{array}$ & $\begin{array}{c}3.16 \\
(0.691)\end{array}$ & $\begin{array}{c}8.5 \\
(0.702)\end{array}$ \\
\hline $\begin{array}{l}\mathrm{T}_{6} \text { : Butachlor } 1.0 \mathrm{~kg} \text { a. i. ha }{ }^{-1} \text { as PPI with two intercultural operations at } 30 \text { and } 45 \text { DAS followed by } \\
\text { one hand weeding at } 21 \text { DAS }\end{array}$ & $\begin{array}{c}7.66 \\
(0.725)\end{array}$ & $\begin{array}{c}3.0 \\
(0.689)\end{array}$ & $\begin{array}{l}10.66 \\
(0.725)\end{array}$ \\
\hline $\begin{array}{l}\text { T7: Sole crop of non Bt cotton C. var. LRA } 5166 \text { with recommended cultural weed management i.e. } \\
\text { Three intercultural operations at 21, } 42 \text { and } 63 \text { DAS followed by two hand weedings at } 21 \text { and } 42 \\
\text { DAS. }\end{array}$ & $\begin{array}{c}7.83 \\
(0.728)\end{array}$ & $\begin{array}{c}2.0 \\
(0.680)\end{array}$ & $\begin{array}{c}9.83 \\
(0.713)\end{array}$ \\
\hline $\begin{array}{l}T_{8} \text { : Sole crop of soybean C. var. Punjab-1 with two intercultural operations at } 21,42 \text { DAS followed } \\
\text { by two hand weedings at } 21 \text { and } 42 \text { DAS. }\end{array}$ & $\begin{array}{c}7.33 \\
(0.745)\end{array}$ & $\begin{array}{c}5.3 \\
(0.709)\end{array}$ & $\begin{array}{l}11.16 \\
(0.724)\end{array}$ \\
\hline $\mathrm{SED} \pm 5 \%$ & & 0.028 & 0.013 \\
\hline $\mathrm{CD} \pm \overline{5} \%$ & 0.040 & NS & NS \\
\hline
\end{tabular}

Table.6 Weed biomass $\mathrm{g} \mathrm{M}^{2}$ in third season.

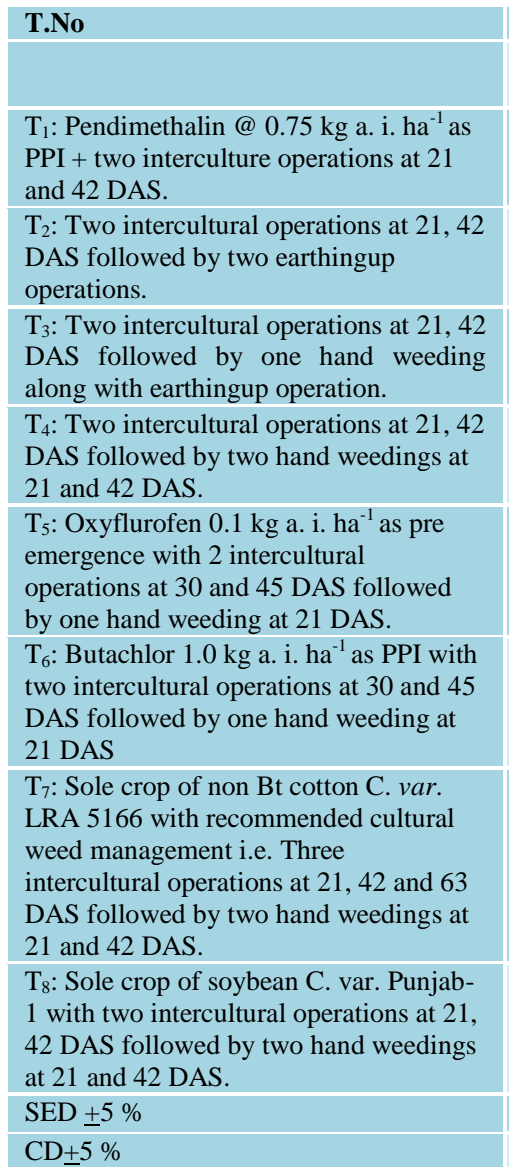

\begin{tabular}{|c|c|c|c|c|c|c|c|c|}
\hline \multicolumn{3}{|c|}{$30^{\text {th }}$ June. } & \multicolumn{3}{|c|}{$26^{\text {th }}$. July. } & \multicolumn{3}{|c|}{$9^{\text {th }}$ August. } \\
\hline $\begin{array}{l}\text { Inter } \\
\text { culture }\end{array}$ & $\begin{array}{l}\text { Hand } \\
\text { weeding }\end{array}$ & Total & $\begin{array}{l}\text { Inter } \\
\text { culture }\end{array}$ & $\begin{array}{l}\text { Hand } \\
\text { weeding }\end{array}$ & Total & $\begin{array}{l}\text { Inter } \\
\text { culture }\end{array}$ & $\begin{array}{l}\text { Hand } \\
\text { weeding }\end{array}$ & Total \\
\hline 4.13 & & 4.13 & & 1.33 & 1.33 & 1.88 & 2.78 & 4.66 \\
\hline 18 & & 18 & 2.16 & 1.16 & 3.33 & 1.33 & 2.94 & 4.27 \\
\hline 2.67 & & 2.67 & 3.33 & 1.83 & 5.16 & 1.55 & 2.22 & 3.77 \\
\hline 5.3 & 8.1 & 13.4 & 3.33 & 2 & 5.33 & 1.66 & 1.67 & 3.33 \\
\hline 1.17 & 8.77 & 9.93 & 2.16 & 5 & 5 & 1 & 0.56 & 1.56 \\
\hline & & & 4.33 & 2.33 & 6.66 & 0.89 & 0.44 & 1.33 \\
\hline 6.37 & 9.13 & 15.83 & 2.83 & 1.83 & 4.66 & 1.66 & 2 & 3.66 \\
\hline 6.8 & 8.3 & 15.1 & 2.33 & 3 & 5.33 & 2.44 & 2.22 & 4.66 \\
\hline & & & 0.93 & 1.58 & 1 & 0.38 & & \\
\hline & & 7.69 & NS & NS & NS & NS & 0.89 & \\
\hline
\end{tabular}

*Figures in parenthesis are $\log \mathrm{V}(\mathrm{x}+21)$ 
Table.7 LER values by weed management strategies

\begin{tabular}{|c|c|c|c|c|c|c|c|c|c|c|c|c|}
\hline \multirow[t]{2}{*}{ Tr. No } & \multicolumn{4}{|c|}{ Seed cotton yields $\mathrm{Kg} \mathrm{ha}^{-1}$} & \multicolumn{4}{|c|}{ Soybean yield $\mathrm{Kg} \mathrm{ha}^{-1}$} & \multicolumn{4}{|c|}{ LER values for intercropping } \\
\hline & $\begin{array}{c}\text { Year } \\
\text { I }\end{array}$ & $\begin{array}{c}\text { Year } \\
\text { II }\end{array}$ & $\begin{array}{c}\text { Year } \\
\text { III }\end{array}$ & Mean & $\begin{array}{c}\text { Year } \\
\text { I }\end{array}$ & $\begin{array}{c}\text { Year } \\
\text { II }\end{array}$ & $\begin{array}{c}\text { Year } \\
\text { III }\end{array}$ & Mean & $\begin{array}{c}\text { Year } \\
\text { I }\end{array}$ & $\begin{array}{c}\text { Year } \\
\text { II }\end{array}$ & $\begin{array}{c}\text { Year } \\
\text { III }\end{array}$ & Mean \\
\hline $\begin{array}{l}\mathrm{T}_{1} \text { : Pendimethalin @ } 0.75 \mathrm{~kg} \mathrm{a.} \mathrm{i.} \mathrm{ha} \mathrm{h}^{-1} \text { as } \\
\text { PPI + two interculture operations at } 21 \text { and } \\
42 \text { DAS. }\end{array}$ & 1061 & 257 & 421 & 580 & 1675 & 661 & 578 & 971 & 2.05 & 1.22 & 2.36 & 1.88 \\
\hline $\begin{array}{l}\mathrm{T}_{2} \text { : Two intercultural operations at } 21,42 \\
\text { DAS followed by two earthingup } \\
\text { operations. }\end{array}$ & 978 & 217 & 257 & 484 & 1360 & 595 & 793 & 916 & 1.92 & 1.09 & 1.91 & 1.64 \\
\hline $\begin{array}{l}\mathrm{T}_{3} \text { : Two intercultural operations at } 21,42 \\
\text { DAS followed by one hand weeding along } \\
\text { with earthingup operation. }\end{array}$ & 1048 & 228 & 271 & 515 & 1551 & 660 & 811 & 1007 & 2.07 & 1.20 & 2.01 & 1.76 \\
\hline $\begin{array}{l}T_{4} \text { : Two intercultural operations at } 21,42 \\
\text { DAS followed by two hand weedings at } 21 \\
\text { and } 42 \text { DAS. }\end{array}$ & 1072 & 254 & 258 & 528 & 1532 & 708 & 869 & 1036 & 2.16 & 1.29 & 2.06 & 1.84 \\
\hline $\begin{array}{l}\mathrm{T}_{5} \text { : Oxyflurofen } 0.1 \mathrm{~kg} \text { a. i. ha }{ }^{-1} \text { as pre } \\
\text { emergence with } 2 \text { intercultural operations at } \\
30 \text { and } 45 \text { DAS followed by one hand } \\
\text { weeding at } 21 \text { DAS. }\end{array}$ & 1073 & 427 & 254 & 585 & 1293 & 797 & 811 & 967 & 1.98 & 1.58 & 1.97 & 1.84 \\
\hline $\begin{array}{l}\mathrm{T}_{6} \text { : Butachlor } 1.0 \mathrm{~kg} \text { a. i. ha }{ }^{-1} \text { as PPI with } \\
\text { two intercultural operations at } 30 \text { and } 45 \\
\text { DAS followed by one hand weeding at } 21 \\
\text { DAS }\end{array}$ & - & & 256 & 256 & & & 813 & 813 & & & 1.98 & 1.98 \\
\hline $\begin{array}{l}\mathrm{T}_{7} \text { : Sole crop of non Bt cotton C. var. LRA } \\
5166 \text { with recommended cultural weed } \\
\text { management i.e. Three intercultural } \\
\text { operations at } 21,42 \text { and } 63 \text { DAS followed } \\
\text { by two hand weedings at } 21 \text { and } 42 \text { DAS }\end{array}$ & 1083 & 1171 & 251 & 835 & & & & & & & & \\
\hline $\begin{array}{l}\mathrm{T}_{8} \text { : Sole crop of soybean C. var. Punjab-1 } \\
\text { with two intercultural operations at } 21,42 \\
\text { DAS followed by two hand weedings at } 21 \\
\text { and } 42 \text { DAS. }\end{array}$ & - & & & & 1309 & 654 & 876 & 946 & 1 & 1 & 1 & 1 \\
\hline $\mathrm{SED} \pm 5 \%$ & 67.0 & & & & 98.8 & 62.7 & 94.1 & & & & & \\
\hline $\mathrm{CD} \pm 5 \%$ & & 176 & 101 & & & & & & 0.24 & 0.12 & 0.5 & \\
\hline
\end{tabular}

Table.8 Profitability of cotton-soybean intercropping system with herbicides

\begin{tabular}{|c|c|c|c|c|c|c|c|c|}
\hline \multirow[t]{2}{*}{ Tr. No } & \multicolumn{4}{|c|}{ Weed management cost Rs ha $^{-1}$} & \multicolumn{4}{|c|}{ B:C ratio } \\
\hline & Year I & Year II & Year III & Mean & Year I & Year II & Year III & Mean \\
\hline $\begin{array}{l}\mathrm{T}_{3} \text { : Two intercultural operations at } 21,42 \text { DAS followed by one } \\
\text { hand weeding along with earthingup operation. }\end{array}$ & 801 & 1148 & 937 & 962 & 3.41 & 1.44 & 1.46 & 2.10 \\
\hline $\begin{array}{l}\mathrm{T}_{4} \text { : Two intercultural operations at } 21,42 \mathrm{DAS} \text { followed by two } \\
\text { hand weedings at } 21 \text { and } 42 \text { DAS. }\end{array}$ & 2467 & 1961 & 1087 & 1838 & 4.16 & 1.66 & 1.5 & 2.44 \\
\hline $\begin{array}{l}\mathrm{T}_{6} \text { : Butachlor } 1.0 \mathrm{~kg} \text { a. i. ha }{ }^{-1} \text { as PPI with two intercultural } \\
\text { operations at } 30 \text { and } 45 \text { DAS followed by one hand weeding at } 21 \\
\text { DAS }\end{array}$ & & & 861 & 861 & & & 1.34 & 1.34 \\
\hline $\begin{array}{l}\mathrm{T}_{7} \text { : Sole crop of non Bt cotton C. var. LRA } 5166 \text { with } \\
\text { recommended cultural weed management i.e. Three intercultural } \\
\text { operations at } 21,42 \text { and } 63 \text { DAS followed by two hand weedings } \\
\text { at } 21 \text { and } 42 \text { DAS. }\end{array}$ & 2853 & 2075 & 1146 & 2024 & 2.18 & 3.93 & 0.53 & 2.21 \\
\hline $\mathrm{CD} \pm 5 \%$ & 1053 & 309 & 193 & & & & & \\
\hline
\end{tabular}




\section{Seed cotton and soybean grain yields}

The results found in three seasons highest LER of 2.16, 1.58 and 2.36 were observed, the lowest value of 1.58 was due to excess rains caused excess vegetative growth of soybean reduced seed cotton yield of intercropping. Despite these draw backs for both the crops a mean LER of 1.98 was observed over 3 years which clearly indicates the profitability of cotton + soybean intercropping with reasonably acceptable weed management. The seed cotton and soybean grain yields were non significantly influenced in first season due to efficient distribution of rainfall non $\mathrm{Bt}$ cotton compensated from its reduction in growth. In $2^{\text {nd }}$ season due to intercropping soybean yields were non significantly reduced but in non $\mathrm{Bt}$ cotton significant reduction in yields were observed compared to sole non Bt cotton but the weed management treatments remained at non-significant level due to higher growth of soybean under excess rains. In $3^{\text {rd }}$ season also soybean results due to intercropping were unaffected but seed cotton yield significantly improved with the Pendimethalin herbicide @ $0.75 \mathrm{~kg}$ a. i. ha ${ }^{-1}$ as P.P.I. +2 intercultural operations at 21 and 42 DAS. LER values also indicates in treatment $\mathrm{T}_{2}$ (2 intercultural operations at 21, 42 DAS followed by two earthingup operations) significant differences were observed due to earthingup operations which did not suppressed the weeds under excess rains, whereas Oxyflurofen $0.1 \mathrm{~kg}$ a. i. $\mathrm{ha}^{-1}$ as pre emergence with 2 intercultural operations at 30 and 45 DAS followed by one hand weeding at 21 DAS to remove resistant broad leaved weeds significantly outperformed over farmer's practice in $2^{\text {nd }}$ season. LER values in $3^{\text {rd }}$ season were significantly higher than sole crops but among weed management treatments differences remains at nonsignificant levels. The economic indicators like $\mathrm{C}: \mathrm{B}$ ratio on average over three years suggested Oxyflurofen $0.1 \mathrm{~kg}$ a. i. ha ${ }^{-1}$ as pre emergence and two intercultures and hand weedings at 21 and 42 DAS are most economical followed by Pendimethalin herbicide@ $0.75 \mathrm{~kg}$ a. i. ha ${ }^{-1}$ as PPI in non Bt cotton-soybean intercropping with +2 interculture operations at 21 and 42 DAS Butachlor@0.75 kg a. i. ha ${ }^{-1}$ as PPI was also evaluated in one year which was also economical in non $\mathrm{Bt}$ cotton-soybean intercropping. Butachlor field efficacy was further confirmed from other field studies at this institute which found it was equally effective. The results were in conformity with Nimbole, 1990 and Ramesh Babu and Rao, 1993.

Therefore the economic and effective integrated weed management packages were recommended for non $\mathrm{Bt}$ cotton-soybean intercropping involving low cost herbicides such as Oxyflurofen @ $0.1 \mathrm{~kg}$ a. i. ha ${ }^{-1}$ as pre emergence and Butachlor $1.0 \mathrm{~kg}$ a. i. ha ${ }^{-1}$ as PPI along with two interculture operations at 21 and 42 days after sowing and if any resistant weeds are there which can be removed by hand weeding.

\section{Acknowledgement}

The financial assistance received under World Bank funded IPM programme through Govt. of India is duly acknowledged by the Author.

\section{References}

Anonymous, 1994: Evaluation of suitable soybean genotypes for maximizing resource use efficiency in intercropped cotton. Annual report of Central Institute for Cotton Research, Nagpur, PP.37.

Nimble, N. N., 1990: Chemical weed control in relation to intercropping of rainfed hybrid cotton. Annals of agricultural research, 11(1):106-108. 
Raju, Ravinder, A., Thakare, Soniya, Majumdar, G., Bharambe, P., R. 2013. Risk aversion in shallow soils with innovative intercropping systems Journal of Cotton Research and Development 27(1): 37-44.

Ramesh Babu and Rao, M.C.H., 1993: Evaluation of herbicides for weed control in irrigated cotton integrated weed management for sustainable agriculture. Proc. Indian Society for weed Science International symposium Hissar, $18^{\text {th }}-20^{\text {th }}$. November, 1993 Vol. 1: 129-131.

Ravinder Raju, A. and Soniya Thakare, 2014: Profitability and FUE of Bt cotton based Intercropping systems under changing rainfall patterns in Vertisols of India. African Journal of Agricultural Research 8(24):3177-3185

\section{How to cite this article:}

Ravinder Raju, A. 2018. Rice Herbicides for Cotton + Soybean Intercropping. Int.J.Curr.Microbiol.App.Sci. 7(08): 4427-4435. doi: https://doi.org/10.20546/ijcmas.2018.708.467 